\title{
Das Europäische Sicherheits- und Verteidigungskolleg Wesentliches Instrument zur Weiterentwicklung der Europäischen Sicherheits- und Verteidigungspolitik
}

\author{
Hans-Bernhard Weißerth*
}

\begin{abstract}
In 1999 the Treaty of Amsterdam came into force and the needed institutional preconditions and bodies for an effective Common Security and Defence Policy had been established. Up to now we can see impressive improvements and a lot of European missions in the field of a Common Foreign and Security Policy. But success and efficiency don't depend only on these decisions. There is a need for personnel with an understanding and expertise to act on the basis of a common security culture. In 2005 the European Council decided to establish the European Security and Defence College (ESDC). Organisation, training, and perspectives for he future development of the college are described in detail.
\end{abstract}

Keywords: Europäisches Sicherheits- und Verteidigungskolleg, europäische Sicherheitskultur, Europäische Sicherheits- und Verteidigungspolitik

$\mathrm{M}$ it Bulgarien und Rumänien traten am 1. Januar 2007 zwei neue Mitgliedstaaten der Europäischen Union bei. Wie schon die letzte große Erweiterung im Mai 2002 mit zehn neuen Mitgliedstaaten kann auch dieser kleinere Erweiterungsschritt als historisches Ereignis betrachtet werden. 27 souveräne Staaten sind nun im Rahmen einer friedlichen Integration in der Union zusammengeschlossen. Hierdurch wird einmal mehr der wesentliche Daseinszweck der Europäischen Union demonstriert - das Zusammenleben der Bürger Europas in Frieden und Wohlstand. Die erweiterte Union zählt nun an die 500 Millionen Bürger. Die Bevölkerung ist damit beinahe doppelt so groß wie die der Vereinigten Staaten. Die Wirtschaftskraft der Europäischen Union entspricht einem Viertel des weltweiten Bruttosozialprodukts. Wir sind einer der wichtigsten Wirtschafts- und Handelspartner und wir geben mit Abstand die meiste Entwicklungshilfe. Mit unserer Größe und unserer Wirtschaftskraft haben wir - entsprechend unserer durch die Staats- und Regierungschefs im Dezember 2003 verabschiedeten Sicherheitsstrategie - als strategischer und globaler Akteur eine Verantwortung in der ganzen Welt.

Neben der Erweiterung wird ein erfolgreicher Abschluss eines Verfassungsvertrages für Europa ein weiterer wichtiger Meilenstein für die Europäische Union sein. Wie die Aussichten für eine Ratifizierung des Vertrages durch alle Mitgliedstaaten ${ }^{1}$ zu beurteilen sind, wird sich bis zum Ende der Deutschen EURatspräsidentschaft im Juni diesen Jahres zeigen, wenn - mit Blick auf 2009, das Jahr für die nächsten Wahlen zum Europäischen Parlament - zumindest ein Fahrplan für die Behandlung und die weitere Ratifizierung des Verfassungswerkes vorliegen soll. Solange werden sich die 27 Mitgliedstaaten auf absehbare Zeit bei ihrer Arbeit weiterhin auf die bestehenden Verträge stützen müssen.

\footnotetext{
* Hans-Bernhard Weißerth (Dipl.-Päd.) ist seit 1999 Mitarbeiter im Politischen Stab des Hohen Repräsentanten für die Gemeinsame Außen- und Sicherheitspolitik im Rat der Europäischen Union, seit 2004 Leiter der ESVP Task Force und verantwortlich für das Funktionieren und die Weiterentwicklung des Europäischen Sicherheits- und Verteidigungskollegs.

118 der 27 Mitgliedstaaten haben den Verfassungsvertrag in seiner vorliegenden Form bereits ratifiziert! In Frankreich und den Niederlanden wurde der Entwurf in nationalen Referenda mehrheitlich abgelehnt.
}

\section{Die Entwicklung der gemeinsamen Außen-, Sicherheits- und Verteidigungspolitik}

Was die Gemeinsame Außen- und Sicherheitspolitik (GASP) betrifft, stellt dies die Union nicht vor unüberbrückbare Probleme. Der Vertrag von Nizza enthält, was die praktische und funktionale Seite betrifft, bereits vieles, wodurch die Herausforderungen der Erweiterung bewältigt, die Organe auch in der erweiterten Union wirksam funktionieren und die Beschlussfassungsverfahren gestrafft werden. Hinzu kommt, dass in den letzten Jahren die GASP eine fast unvergleichbare Dynamik entfalten und sich bis heute zu einem wesentlichen Motor der Integration entwickeln konnte. Die GASP ist das Integrationsprojekt, das - so die Meinungsumfragen - von den Bürgern am meisten getragen, ja sogar ausdrücklich erwünscht ist. Auch der politische Wille der verantwortlichen Politiker scheint - bei aller Euroskepsis - vorhanden zu sein. Sie haben erkannt, dass Europa nur dann eine Rolle spielen kann, wenn es einig ist. Nur mit europäischen Lösungen und darüber hinaus nur in Zusammenarbeit mit den wichtigsten Partnern, insbesondere den USA, kann die Sicherheit der europäischen Bürger gewährleistet werden.

In den letzten Jahren haben wir erhebliche Fortschritte erzielt. Seit dem Inkrafttreten des Vertrages von Amsterdam im Herbst 1999 wurden zunächst die institutionellen Voraussetzungen und Gremien geschaffen, die für eine wirksame GASP erforderlich sind. Parallel dazu wurden umfassende Prozesse eingeleitet zur Entwicklung glaubwürdiger militärischer und ziviler Fähigkeiten, die für ein erfolgreiches internationales Krisenmanagement der Europäischen Union unumgänglich sind. Die EU-NATO-Vereinbarungen erlauben den Rückgriff auf NATO-Mittel und -Fähigkeiten, verstärken damit die operationellen Fähigkeiten der Union und vermeiden gleichzeitig unnötige Duplizierungen im militärischen Bereich. Mit der vom Europäischen Rat gebilligten Europäischen Sicherheitsstrategie haben wir eine bemerkenswerte Übereinstimmung zwischen 27 europäischen Staaten in wesentlichen Fragen der europäischen Sicherheit festgestellt. Diese Sicherheitsstrategie bildet einen wichtigen Bezugspunkt sowohl für langfristige 
europäische Strategien als auch für aktuelle politische Probleme einschließlich der Weiterentwicklung der Europäischen Sicherheits- und Verteidigungspolitik (ESVP).

Was konkretes Krisenmanagement betrifft, haben die Europäer seit 2003 in zahlreichen zivilen und militärischen Operationen unter Beweis gestellt, dass sie es mit der Entwicklung ihrer Gemeinsamen Außen- und Sicherheitspolitik und Verteidigungspolitik ernst meinen. Auf dem Balkan wurde die EU zum wichtigsten Akteur, als Mitglied des »Quartetts « ist sie aktiv engagiert bei der Suche nach Lösungsmöglichkeiten für den Nahostkonflikt. Sie ist engagiert in Afghanistan, dem Nahen Osten, dem Südkaukasus und in Afrika. Mit ihrer neu konzipierten Nachbarschaftspolitik verstärkt die EU ihren Einsatz in ihrer unmittelbaren Nachbarschaft im Süden und Osten, um durch eine Stabilisierung dieser Regionen auch zu einer Erhöhung der Sicherheit der EU beizutragen. Dabei ist die Zusammenarbeit der Union mit anderen internationalen Organisationen, insbesondere den Vereinten Nationen, von wesentlicher Bedeutung und auch Beweis dafür, dass nur mit einem »effektiven Multilateralismus « (eine der Kernaussagen der Sicherheitsstrategie) den Risiken und Bedrohungen von heute erfolgreich begegnet werden kann.

Mit einer erfolgreichen Verabschiedung des Verfassungsvertrages in seiner vorliegenden Form würden neue wichtige Entscheidungen getroffen und Weichen gestellt, die der GASP und der ESVP einen weiteren Schub geben würden. Im außenpolitischen Bereich ist die Schaffung eines Außenministers der Union und - damit einhergehend - die Einrichtung eines europäischen Auswärtigen Dienstes von herausragender Bedeutung. Aus verteidigungspolitischer Sicht wird sich die bereits begonnene Arbeit der Europäischen Verteidigungsagentur positiv auf die Rüstungskooperation der Mitgliedstaaten auswirken, und eine »permanent strukturierte Zusammenarbeit « würde endlich eine bessere Plattform für die Mitgliedstaaten schaffen, die bereit sind, mehr Integration im militärischen Bereich zu wagen und dabei EU-Strukturen zu nutzen.

\section{Eine erfolgreiche GASP und ESVP - auch eine Frage der Ausbildung}

Erfolg und Effizienz der GASP und ESVP hängen aber nicht allein von diesen signifikanten Entscheidungen und Richtungsvorgaben ab. Die GASP/ESVP-Strukturen und -Gremien, die zivilen, militärischen und zivil-militärischen Stäbe, die zuständigen nationalen Ministerien und die Delegationen in Brüssel, auch die parlamentarischen Gremien, brauchen Personal, das das Verständnis und die notwendige Expertise besitzt, im Sinne einer gemeinsamen Sicherheitskultur effektiv und effizient zu handeln. Javier Solana als Hoher Vertreter für die GASP unterstreicht von Beginn seiner Amtszeit an die Notwendigkeit und Bedeutung der Herausbildung einer gemeinsamen europäischen Sicherheitskultur innerhalb der ESVP.

Praktische Unterstützung erhielt diese Sichtweise durch verschiedene Initiativen im Rat der Europäischen Union, der Europäischen Kommission und in den Mitgliedstaaten. Dazu gehören insbesondere:

- die Gründung des Europäischen Polizeikollegs (CEPOL),
- das Ausbildungsprojekt der Europäischen Kommission im Bereich des zivilen Krisenmanagements (European Group on Training/EGT) mit Trainingkursen in den Bereichen »Rule of Law « und »Civil Administration«,

- die Etablierung des »European Diplomatic Programme« (EDP) im Bereich der diplomatischen Ausbildung, eine Kompromisslösung für einen Vorschlag, vor dem Hintergrund einer sich entwickelnden GASP, eine Europäische Diplomatische Akademie aufzubauen,

- der Vorschlag für den Aufbau eines Europäischen Sicherheits- und Verteidigungskollegs (ESDC). ${ }^{2}$

Die Schaffung einer gemeinsamen Ausbildung im Bereich der ESVP zur Unterstützung der Entwicklung einer gemeinsamen Sicherheitskultur geht im Wesentlichen auf die Initiative der griechischen Ratspräsidentschaft im Jahre 2002 zurück. Dieser Initiative folgend verabschiedete der Rat der Europäischen Union im November 2003 die »EU-Ausbildungspolitik im Bereich der ESVP « und im September 2004 das »EU-Trainingkonzept im Bereich der ESVP«, zwei Dokumente, die fortan die Richtung für praktische Schritte im Bereich der Ausbildung vorgeben und insbesondere das Ziel verfolgen, die verschiedenen Initiativen auf europäischer und nationaler Ebene besser zu koordinieren. Alle Akteure, die Ausbildung im Bereich der ESVP betreiben (die einzelnen Mitgliedstaaten und die europäischen Akteure wie ESDC, CEPOL, EDP, EGT), werden damit in den jährlichen EU-Ausbildungszyklus eingebunden, der mit der Analyse des Ausbildungsbedarfs beginnt, und darauf aufbauend, die Erstellung des jährlichen EU-Ausbildungsprogramms, die Durchführung der Ausbildung durch die europäischen und nationalen Institute und deren abschließende Auswertung einschließt. Mit dem jährlichen Erfahrungsbericht des Politischen und Sicherheitspolitischen Komitees (PSK) wird dieser jährliche Ausbildungszyklus abgeschlossen. Dem Rat der Europäischen Union werden dabei Vorschläge zur Verbesserung der Ausbildung im Bereich der ESVP zur Entscheidung vorgelegt.

Ein zusätzlicher und nicht unwesentlicher Aspekt dieses Konzepts ist die Zusammenarbeit mit internationalen Organisationen, insbesondere mit den Vereinten Nationen, der NATO und der Afrikanischen Union, um eine bessere Grundlage für eine enge Kooperation im internationalen Krisenmanagement zu schaffen.

\section{Auftrag und Zielsetzung des Europäischen Sicherheits- und Verteidigungskollegs}

Das EU-Ausbildungskonzept im Bereich der ESVP macht deutlich, dass das Europäische Sicherheits- und Verteidigungskolleg ein Element eines umfassenderen Ansatzes ist. Während die meisten Akteure Ausbildung mit Schwerpunkt auf der operationellen und taktischen Ebene durchführen, zielt die Arbeit des Kollegs bewusst auf die strategische Ebene der Ausbildung, auf die gemeinsame Ausbildung des zivilen und militärischen Personals in enger Anbindung an die EU-Organe. Dies macht das Kolleg bereits heute zum entscheidenden Akteur für die Ausbildung im Bereich der ESVP. 2 Deutsch-französische Initiative, offiziell vorgeschlagen beim Vierer-Gipfel
zwischen Belgien, Deutschland, Frankreich und Luxemburg am 29. April 2003 in Brüssel. 
Dem Vorschlag einiger Mitgliedstaaten folgend, beschloss der Rat der Europäischen Union am 18. Juni 2005 die Gemeinsame Aktion zur Einrichtung eines Europäischen Sicherheitsund Verteidigungskollegs. Auf dieser Grundlage funktioniert das Kolleg heute als Netzwerk, das nationale Institute, Kollegs, Akademien und andere Stellen, die sich innerhalb der EU mit Fragen der Sicherheits- und Verteidigungspolitik befassen, sowie das Institut der Europäischen Union für Sicherheitsstudien in Paris umfasst. Es pflegt enge Verbindungen zu den relevanten EU-Organen. Hauptauftrag ist die Vermittlung von Ausbildungsmaßnahmen im Bereich der ESVP auf strategischer Ebene, um ein gemeinsames Verständnis der ESVP bei zivilem und militärischem Personal zu entwickeln und zu fördern und um über diese Maßnahmen in Bezug auf verschiedene Aspekte der ESVP bewährte Verfahren zu ermitteln und zu verbreiten.

Durch die Ausbildungsmaßnahmen verfolgt das Europäische Sicherheits- und Verteidigungskolleg die folgenden Zielsetzungen:

- die weitere Festigung der europäischen Sicherheitskultur im Rahmen der ESVP;

- die Förderung eines besseren Verständnisses der ESVP als wesentliches Element der GASP;

- die Ausstattung der EU-Stellen mit sachkundigem Personal, das alle ESVP-Themen effizient bearbeiten kann;

- die Ausstattung der Verwaltungen der Mitgliedstaaten mit sachkundigem Personal, das mit der Politik, den Institutionen und den Verfahren der EU vertraut ist;

- zur Förderung beruflicher Beziehungen und Kontakte zwischen den Ausbildungsteilnehmern beizutragen.

\section{Organisation und Finanzierung des Kollegs}

Durch die Gemeinsame Aktion des Rates werden für das Funktionieren des Kollegnetzwerkes die folgenden drei Gremien eingerichtet: ein Lenkungsausschuss, ein Akademischer Exekutivrat und ein kleines, aber permanentes Sekretariat.

Der Lenkungsausschuss, bestehend aus einem Vertreter aus jedem Mitgliedstaat, ist verantwortlich für die Gesamtkoordination und Leitung der Ausbildungsmaßnahmen des Kollegs. Dazu gehören die Erstellung des jährlichen akademischen Programms und der Rahmenlehrpläne für alle Ausbildungsmaßnahmen des Kollegs und die Auswahl der nationalen Institute, in denen Ausbildungskurse stattfinden sollen. Zudem hat der Lenkungsausschuss einen jährlichen Auswertungsbericht den relevanten Ratsgremien vorzulegen.

Der Akademische Exekutivrat ist für die Qualität und Kohärenz der Ausbildungsmaßnahmen verantwortlich. Dazu gehören insbesondere die Ausarbeitung der detaillierten Lehrpläne für alle Ausbildungsmaßnahmen, die Koordination zwischen den einzelnen nationalen Instituten, die Sicherstellung und Überprüfung einheitlicher Standards für die Ausbildung und die Durchführung einer detaillierten Auswertung der Ausbildung.

Das Generalsekretariat des Rates fungiert als Sekretariat des Kollegs. Es unterstützt im Wesentlichen den Lenkungsausschuss und den Akademischen Exekutivrat sowie die Durchführung der Ausbildungsmaßnahmen und arbeitet dabei eng mit der Europäischen Kommission und dem Sekretariat des Europäischen Parlaments zusammen.

Was die Finanzierung betrifft, tragen alle am Kollegnetzwerk beteiligten Mitgliedstaaten, EU-Organe, EU-Agenturen und -Einrichtungen sämtliche Kosten, die im Zusammenhang mit ihrer Beteiligung am Kolleg anfallen, einschließlich Gehältern, Vergütungen, Reisekosten und Kosten in Verbindung mit der organisatorischen und administrativen Unterstützung der Ausbildungsmaßnahmen des Kollegs.

\section{Die Ausbildung des Europäischen Sicherheits- und Verteidigungskollegs}

Die Ausbildungsmaßnahmen des Kollegs umfassen heute konkret zwei verschiedene Kurse, einen einwöchigen ESVP-Grundkurs (ESDP Orientation Course) und einen längeren, modular aufgebauten Kurs auf hohem Niveau (ESDP High Level Course), deren detaillierte Programme auf der Grundlage der Erfahrungen von verschiedenen Pilotprojekten in den Jahren 2003 bis 2005 entwickelt wurden. Darüber hinaus kann der Lenkungsausschuss weitere geeignete Ausbildungsmaßnahmen einleiten. Mit dem Beschluss, einen ESVP-Pilotkurs für Pressesprecher der EU-Institutionen und nationalen Ministerien durchzuführen, wurde erstmals im Herbst 2006 von diesem Passus in der Gemeinsamen Aktion Gebrauch gemacht.

Für das Akademische Jahr 2006/2007 (September 2006 bis August 2007) sind die folgenden Ausbildungsmaßnahmen geplant bzw. bereits durchgeführt oder noch in der Durchführung:

1. ein ESVP-Kurs auf hohem Niveau (ESDP High Level Course) mit fünf Modulen (Dauer jeweils eine Woche) in

- Brüssel im September 2006; Hauptthema: Geschichtliche Entwicklung und Überblick über den derzeitigen Sachstand der GASP und der ESVP; Gastgeber: Belgische Verteidigungsakademie in Zusammenarbeit mit dem Ratssekretariat und der Europäischen Kommission.

- Paris im Oktober 2006; Hauptthema: Entwicklung europäischer Fähigkeiten zum Krisenmanagement; Gastgeber: das Höhere Verteidigungsinstitut Frankreichs.

- Athen im November 2006; Hauptthema: das operationelle Engagement der Europäischen Union im zivilen und militärischen Krisenmanagement; Gastgeber: die griechische Diplomatische Akademie in Zusammenarbeit mit dem griechischen Verteidigungskolleg.

- Madrid im Februar 2007; Hauptthema: die ESVP im regionalen Kontext; Gastgeber: das Höhere Verteidigungsinstitut Spaniens in Zusammenarbeit mit der polnischen Verteidigungsakademie.

- Berlin im März 2007; Hauptthema: zukünftige Perspektiven der ESVP- Entwicklung; Gastgeber: die Bundesakademie für Sicherheitspolitik.

2. ein Pilot-ESVP-Kurs für Personal im Presse- und Informationsdienst, insbesondere Pressesprecher (Dauer: zweieinhalb Tage) in Brüssel im November 2006, Gastgeber: die österreichische Delegation in enger Zusammenarbeit mit dem Ratssekretariat; 
3. ein ESVP-Orientierungskurs in Brüssel im Dezember 2006 (Dauer: eine Woche), Gastgeber: die finnische Präsidentschaft in enger Zusammenarbeit mit dem Ratssekretariat und der Europäischen Kommission;

4. ein ESVP-Orientierungskurs in Brüssel im Februar 2007 (Dauer: eine Woche), Gastgeber: das niederländische Verteidigungskolleg in enger Zusammenarbeit mit dem Ratssekretariat und der Europäischen Kommission;

5. ein ESVP-Orientierungskurs in Brüssel im Mai 2007 (Dauer: eine Woche), Gastgeber: die Bundesakademie für Sicherheitspolitik/Berlin in enger Zusammenarbeit mit dem Ratssekretariat und der Europäischen Kommission.

Im Rahmen der ESVP-Orientierungskurse erhalten die Kursteilnehmer einen Gesamtüberblick über die Entwicklung der ESVP, insbesondere was die folgenden Aspekte betrifft:

- institutionelles Gefüge der EU,

- die Europäische Sicherheitsstrategie: Entwicklung und Implementierung,

- die ESVP-Strukturen und -Verfahren,

- die Entwicklung ziviler und militärischer Fähigkeiten,

- das operationelle Engagement der EU und

- die Zusammenarbeit mit Partnern und internationalen Organisationen.

Der Teilnehmerkreis umfasst grundsätzlich ziviles und militärisches Personal, das sich mit strategischen Aspekten der ESVP befasst. Der Kurs auf hohem Niveau ist besonders für Personal in Führungsfunktionen konzipiert. Jeder Teilnehmer, der einen Lehrgang des Kollegs abschließt, erhält ein vom Hohen Vertreter für die GASP unterzeichnetes Abschlusszertifikat, das von den Mitgliedstaaten bzw. den EU-Institutionen anerkannt wird.

Alle Ausbildungsmaßnahmen stehen den Staatsangehörigen aller Mitgliedstaaten und Beitrittsländer zur Teilnahme offen. Vertreter u.a. von Nichtregierungsorganisationen, akademischen Einrichtungen und der Medien sowie der Wirtschaft können eingeladen werden. Grundsätzlich können auch Staatsangehörige von Bewerberländern und gegebenenfalls von Drittstaaten teilnehmen.

Letzterer Aspekt ist von großer Bedeutung vor dem Hintergrund, dass die Union die ESVP- Entwicklung als offenen und transparenten Prozess betrachtet und im internationalen Krisenmanagement prinzipiell die Beteiligung von Drittstaaten bei ihren Operationen sucht. Soll dies auf allen Ebenen gut funktionieren, setzt dies auch eine gemeinsame Ausbildung voraus. Bereits im letzten Akademischen Jahr 2005/2006, insbesondere während der österreichischen Präsidentschaft, wurden die ersten ESVP-Orientierungskurse für die Beteiligung von Drittstaaten geöffnet, und das mit großem Erfolg. Teilnehmende Kandidatenstaaten und Drittstaaten waren am Beispiel des ESVP-Kurses im Dezember 2006 unter anderem die USA, Kanada, Russland, China, Japan, Thailand, Australien, Norwegen und die Türkei. Auch internationale Organisationen wie die Vereinten Nationen, NATO und die Afrikanische Union waren in diesem Kurs bereits vertreten.
Die bis heute gewonnenen Erfahrungen zeigen, dass die gemeinsame Ausbildung von zivilem und militärischem Personal, von Teilnehmern aus Mitgliedstaaten, Kandidatenstaaten, Drittländern und internationalen Organisationen ein ausgezeichneter Beitrag zur Entwicklung der ESVP und zur Förderung einer gemeinsamen Sicherheitskultur ist. Die Mitgliedstaaten und die EU-Institutionen werden auf diesem Wege Schritt für Schritt mit gut ausgebildetem Personal versorgt, das in der Lage ist, effizient im Bereich der ESVP zu arbeiten. Einschließlich der Pilotkurse und der derzeit laufenden Ausbildungsmaßnahmen, konnten bisher etwa 180 Kursteilnehmer im ESVP-Kurs auf hohem Niveau und etwa 400 Kursteilnehmer in den einwöchigen ESVP- Orientierungskursen ausgebildet werden.

\section{Perspektiven für die Weiterentwicklung des Kollegs}

Dies kann einerseits als ein großer Erfolg des Kollegs gewertet werden, andererseits bleibt angesichts einer schnell wachsenden Nachfrage nach Ausbildungsplätzen für beide Kurse die Ausbildungskapazität des Kollegs insgesamt limitiert, nicht zuletzt aufgrund des bisherigen organisatorischen Ansatzes und nicht ausreichender finanzieller, personeller und infrastruktureller Ressourcen. Soll das Kolleg in Zukunft den steigenden Anforderungen genügen, wird dies in der bisherigen virtuellen Form als Netzwerk ohne eine permanente zentrale Stelle nicht wirklich funktionieren.

Artikel 13 der Gemeinsamen Aktion des Europäischen Rates sieht vor, dass der Lenkungsausschuss einen Bericht über die Tätigkeiten und Perspektiven des Kollegs erarbeitet, in dem auch die finanziellen Modalitäten und das Sekretariat behandelt werden, und diesen Bericht dem Rat bis spätestens 31. Dezember 2007 als Grundlage für eine Überarbeitung der Gemeinsamen Aktion vorlegt. Diese Arbeiten werden im zweiten Halbjahr unter portugiesischer Präsidentschaft stattfinden. Während die Debatte um und die Erarbeitung der Gemeinsamen Aktion im Jahre 2005 noch geprägt war durch eine grundsätzlich ablehnende Haltung einiger Mitgliedstaaten gegenüber der Initiative, bleibt abzuwarten, wie sich - angesichts des großen Erfolgs der bisherigen Ausbildungsmaßnahmen - die Situation zugunsten eines weiteren Ausbaus des Kollegs positiv gewandelt hat. Tatsache ist, dass inzwischen auch Mitgliedstaaten, die der Einrichtung des Kollegs zunächst ablehnend gegenüberstanden, inzwischen zu den Mitgliedstaaten gehören, die regelmäßig nach zusätzlichen Ausbildungsplätzen für ihr Personal fragen. Dies stimmt optimistisch was die zu erwartende zweite Entwicklungsphase des Europäischen Sicherheits- und Verteidigungskollegs betrifft. Zudem wird es auch auf die gesamte politische Stimmungslage im Bereich der GASP und ESVP ankommen. Hier kann entscheidend die Arbeit der deutschen Ratspräsidentschaft beitragen. Je konkreter der Fahrplan und die Maßnahmen für eine erfolgreiche Ratifizierung des Verfassungsvertrages definiert werden können, desto besser wird dies für die politische Stimmungslage in der GASP und ESVP sein, und dies wird erfahrungsgemäß auch direkte Auswirkungen auf die Bereitschaft der Mitgliedstaaten haben, für die weitere Entwicklung und den Aufbau des Kollegs die notwendigen Ressourcen verfügbar zu machen. 